\title{
ON LOCALLY COMPACT METRISABLE SPACES
}

\author{
BY H. E. VAUGHAN*
}

The object of this paper is to present two characterizations, one of the class of locally compact metrisable spaces, the other of the class of locally compact, separable, metrisable spaces (locally compact, perfectly separable Hausdorff spaces), as follows.

THEOREM 1. In order that a metrisable space be locally compact it is necessary and sufficient that it be the difference of two closed sets in every metric space in which it is topologically imbedded. $\dagger$

THEOREM 2. In order that a Hausdorff space be homeomorphic to a totally complete metric space it is necessary and sufficient that it be locally compact and perfectly separable. $\ddagger$

In Theorem 1 the word metric may be replaced, on the one hand, by regular, on the other, by complete metric. This theorem is of interest chiefly because of the similar well known characterizations of the class of all compact metrisable spaces and of the class of all metrisable spaces which are homeomorphic to complete metric spaces. \$ The method of proof also relates it to the two characterizations of the class of compact metrisable

* Alfred H. Lloyd Fellow, University of Michigan.

$\dagger$ A distinction is made between the adjectives metric and metrisable. The word metric implies the definite choice of a metric in a metrisable space. The phrase $A$ is topologically imbedded in $B$ means that $A$ is homeomorphic to a subset of $B$.

$\ddagger$ A totally complete metric space is a metrisable space in which the metric is so chosen that every bounded set is compact. See K. Menger, Über die Dimension von Punktmengen, Monatshefte für Mathematik und Physik, vol. 34 (1926), pp. 135-161; in particular, p. 144.

$\S$ The first of these theorems is obtained by replacing, in Theorem 1 , "locally compact" by "compact" and "the difference of two closed sets" by "closed." The second is obtained by replacing "locally compact" by "homeomorphic to a complete metric space" and "the difference of two closed sets" by "the intersection of a sequence of open sets." A proof of the first may be given along the lines of the proof of the first lemma, stated below. For a proof of the second, see C. Kuratowski, Topologie I, p. 215. 
spaces, as those which are bounded (complete) in every metric.* Theorem 2 is also related to the second theorem mentioned in the footnote above. Its chief interest, however, seems to lie in the fact that the class of spaces characterized was used to a considerable extent by K. Menger $\dagger$ and W. Sierpinski $\ddagger$ but apparently has not before been characterized in non-metric terms.

Theorem 1 is an almost immediate consequence of the following two lemmas.

LEMMA 1. A metrisable space which is locally closed at each of its points $\$$ in every complete metric space in which it is topologically imbedded is locally compact.

Proof. Let $M$ be a metrisable space which is not locally compact. Supposing that a metric has been chosen, there exists a point $a$ of $M$ such that $\| \overline{S(a, 1 / n)}$ is not compact for any positive integer $n$. Consequently, there exists a denumerable family $\left(\left(a_{n i}\right)\right)$ of sequences of points of $M$ such that, for every $i, a_{n i}$ belongs to $S(a, 1 / n)$ and the set $a+\sum_{n, i} a_{n i}$ is a closed subset of $M$. Hence, $\uparrow$ a metric may be chosen for $M$ such that the set $a+\sum_{n, i} a_{n i}$ is isometric to the linear set $(0)+\sum_{n, i}\left(1 / 2^{n}-1 / 2^{n+i}\right)$, where $(x)$ denotes the point whose abscissa is $x$. Now** $M$ may be imbedded isometrically in a complete metric space $\tilde{M}$ and, since each of the sequences $\left(a_{n i}\right)$ now satisfies the Cauchy convergence criterion and all but a finite number of the sequences are contained in any neighborhood of the point $a, M$ is not locally closed at the point $a$ in $\tilde{M}$.

Lemma 2. A locally compact metrisable space is locally closed at each of its points in every Hausdorff space in which it is topologically imbedded.

* See F. Hausdorff, Erweiterung einer Homöomorphie, Fundamenta Mathematicae, vol. 16 (1930), pp. 352-360; in particular, p. 359.

$\dagger$ Loc. cit.

‡ See W. Sierpinski, General Topology, p. 118 et seq.

$\S$ A subset $M$ of a neighborhood space $R$ is said to be locally closed at the point $p$ if there exists a neighborhood of $p$ whose intersection with $M$ is a closed set. See Kuratowski, loc. cit., p. 65.

$\|$ If $a$ is a point of a metric space $M$ and $d$ is a positive real number, $S(a, d)$ denotes the set of all points of $M$ whose distance from $a$ is less than $d$ and $\overline{S(a, d)}$ denotes this set plus its limit points.

I See F. Hausdorff, loc. cit.

** See Kuratowski, loc. cit., p. 200. 
Proof. Each point of a locally compact metrisable space has a neighborhood which is a compact metrisable space. Such a neighborhood is closed in every Hausdorff space in which it is topologically imbedded.

Proof of Theorem 1. This results from the preceding lemmas and the fact that a subset of a regular space which is locally closed at each of its points is the difference of two closed sets, and conversely.*

Proof of Theorem 2. Necessity. Let $M$ be a totally complete metric space. Since every point of $M$ has a bounded neighborhood, $M$ is certainly locally compact, while, since $M=\sum_{1}^{\infty} M_{n}$, where $M_{n}$ is the bounded set of points of $M$ whose distance from some fixed point $a_{0}$ of $M$ is at most $n$, $M$ is the sum of a denumerable family of compact metric spaces and hence is perfectly separable.

Sufficiency. Let $M$ be a locally compact, perfectly separable, Hausdorff space. Then $\dagger$ there exists a perfectly separable compact, Hausdorff space $\tilde{M}$ and a point $a_{0}$ of $\tilde{M}$ such that $M=\tilde{M}-\left(a_{0}\right)$. Since $\tilde{M}$ is a separable metric space it may be considered as a subset of the Hilbert parallelotope $\ddagger$ and its diameter may be assumed to be at most equal to 1 . Let the coordinates of an arbitrary point $x$ of $\tilde{M}$ be denoted by $\left(x_{i}\right)$. Then it may be assumed that $x_{1}=0,\left(0 \leqq x_{i} \leqq 1 / i, i \neq 1\right)$. The sequence $\left(f_{i}^{\prime}(x)\right)$, where $f_{1}^{\prime}(x)=1-d\left(x, a_{0}\right)$ and $f_{i}^{\prime}(x)=x_{i},(i \neq 1)$, is evidently a (1-1) continuous mapping of $\tilde{M}$ on a subset of the Hilbert parallelotope and, since $\tilde{M}$ is compact, is a homeomorphism. The sequence $\left(f_{i}(x)\right)$, where

$$
f_{1}(x)=\frac{f_{1}^{\prime}(x)}{1-f_{1}^{\prime}(x)}, \quad f_{i}(x)=f_{i}^{\prime}(x), \quad(i \neq 1),
$$

is clearly also a homeomorphism of $\tilde{M}-\left(a_{0}\right)$ on a subset of Hil-

* See Kuratowski, loc. cit., p. 65.

† See P. Alexandroff, Über die Metrisation der im kleinen kompakten topologischen Räume, Mathematische Annalen, vol. 92 (1924), pp. 294-301.

$\ddagger$ In this connection, by Hilbert space is meant the metric space whose elements are the infinite sequences, $\left(x_{i}\right)$, of real numbers such that $\sum_{1}^{\infty} x_{i}{ }^{2}<\infty$. The distance, $d(x, y)$, between the points $\left(x_{i}\right)$ and $\left(y_{i}\right)$ is given by the formula $d(x, y)=\left(\sum_{1}^{\infty}\left(x_{i}-y_{i}\right)^{2}\right)^{1 / 2}$. The Hilbert parallelotope is the set of points of Hilbert space such that, for every $i, 0 \leqq x_{i} \leqq 1 / i$. 
bert space. The function $d^{\prime}(x, y)=d(f(x), f(y))$, where $x$ and $y$ are arbitrary points of $M$, may evidently be taken as a metric for $M$ and satisfies the theorem, for a subset of $M$ which is noncompact contains points $x$ such that $f_{1}(x)$ is arbitrarily large.

Corollary. Any locally compact metrisable space is homeomorphic to a metric space in which every bounded, connected, set is compact.

Proof. Let $M$ be a locally compact metrisable space. Then $M$ is a sum of disjoint open sets each of which is a locally compact, separable, metrisable space.* By Theorem 2 , each of these is homeomorphic to a totally complete metric space. The latter may be combined in the following manner to form a metric space of the sort described in the corollary. In each of the above mentioned spaces choose some definite point and call it the origin of the space. Let $M_{1}, M_{2}$, and $M_{3}$ denote any three of the above spaces, let $d_{1}, d_{2}$, and $d_{3}$ denote the respective metrics, and let $o_{1}, o_{2}$, and $o_{3}$ denote the respective origins. If $x$ and $y$ are points of the same space, say $M_{1}$, define $d(x, y)$ as $d_{1}(x, y)$. If $x$ and $y$ belong to different spaces, say $M_{1}$ and $M_{2}$, respectively, define $d(x, y)$ as $d_{1}\left(x, o_{1}\right)+d_{2}\left(y, o_{2}\right)+1$. The function $d(x, y)$ so defined is easily seen to be a metric which defines a space homeomorphic to $M$ in which every bounded connected set is compact.

UNIVERSITY OF MiCHIGAN

* See P. Alexandroff, loc. cit. 